\title{
Rowell syndrome secondary to anti-tubercular therapy: A case report and literature review
}

\section{Bibush Amatya, Leison Maharjan}

\author{
Institute of Medicine, Tribhuvan University Teaching Hospital, Kathmandu, Nepal
}

Corresponding author: Dr. Bibush Amatya, E-mail: doc_bibush@hotmail.com

\begin{abstract}
Rowell syndrome is a condition in which lupus erythematosus is associated with erythema multiformae-like target lesions. The authors describe the condition in a middle-aged woman with drug induced lupus erythematosus and erythema multiformae-like targetoid lesions who initially improved with systemic steroids and antimalarial but succumbed to pulmonary embolism.
\end{abstract}

Key words: Rowell's; Erythema multiformae-like lesions; Drug induced lupus; Pulmonary embolism

\section{INTRODUCTION}

Rowell syndrome is a condition in which lupus erythematosus is associated with erythema multiformaelike target lesions. The presence of targetoid lesions in patients with lupus erythematosus was first described by Scholtz in 1992 [1]. Rowell syndrome was first described by Rowell et al. in 1963 in a study of 120 patients with discoid lupus erythematosus (DLE) out of which four patients had erythema multiformaelike target lesions. The syndrome included lupus erythematosus with erythema multiformae-like targetoid lesions, the presence of ANA positivity in a speckled pattern, rheumatoid factor (RF) positivity and precipitating antibody to a saline extract of human tissue (anti-SJT) [2]. Zeitouni et al. in 2000 introduced major and minor criteria for the diagnosis of Rowell syndrome. According to Zeitouni et al., the presence of all three major criteria and one minor criteria is required for the diagnosis of Rowell syndrome [3].

The three major criteria are:

- Acute, subacute or discoid lupus erythematosus

- Presence of erythema multiformae-like target lesions

- Positivity of ANA in a speckled pattern.
The minor criteria are:

- Chilblains

- Anti-Ro or anti-La positivity

- Positive rheumatoid factor.

There have been around 35 cases of Rowell syndrome described in the literature [4]. Lupus erythematosus can be induced by drugs, with procainamide and hydralazine being the most common culprit agents. Isoniazid, which is one of the anti-tubercular drugs, is also known to induce lupus erythematosus [5].

We describe Rowell's syndrome in a middle-aged lady.

\section{CASE REPORT}

A 40 years old lady presented to our emergency with the complaints of

- Redness and swelling of face for 7 days

- Erosions and hemorrhagic crusting of lips for 5 days

- Painful superficial erosions and ulcers on oral cavity for 5 days.

The lady had been diagnosed with pulmonary tuberculosis two months back based on history of fever and cough for one month, suggestive chest x-ray, sputum acid-fast bacillus stain and Mantoux test

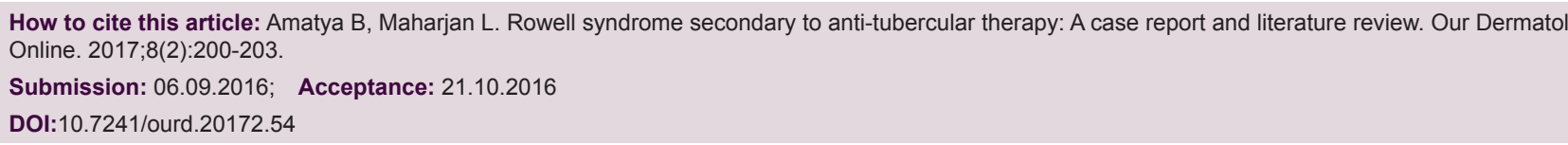


findings two months back and was subsequently put on tablet isoniazid $300 \mathrm{mg}$ once daily, tablet rifampin $450 \mathrm{mg}$ once daily, tablet pyrazinamide $1500 \mathrm{mg}$ once daily and tablet ethambutol $1200 \mathrm{mg}$ once daily for two months. There was no history of a sore throat, joint or muscle pain, pain abdomen, and chilblains. Her bowel and bladder habits were normal.

Her physical examination revealed swelling of her face including perioral and periorbital regions. There were erosions on her lips (Fig. 1).

There were multiple discrete erythematous targetoid lesions (Figs. 2 and 3) and non-blanchable purpura on her back and extremities including palms and soles (Figs. 3-5).

There were no other significant findings on cutaneous and systemic examination.

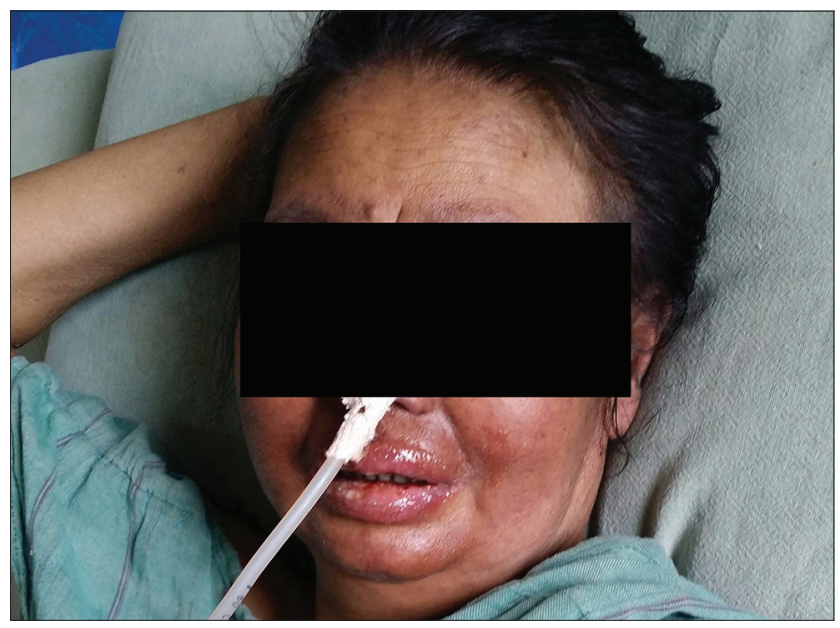

Figure 1: A 40-year-old lady with swelling of her lips.



Figure 2: Multiple discrete purpura and erythematous target-like lesions on the back.
The differentials that had been considered at the time of diagnosis were:

- Stevens-Johnson syndrome secondary to antitubercular therapy

- Drug reaction, eosinophilia and systemic symptoms (DRESS) secondary to anti-tubercular therapy

- Anti-tubercular therapy induced lupus erythematosus

- Erythema multiformae major.

Her investigation reports revealed the following:

- Haemoglobin - $10 \mathrm{~g} / \mathrm{dL}$ (reference value: $12-14 \mathrm{~g} / \mathrm{dL}$ )

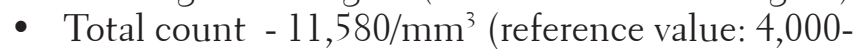
$\left.11,000 / \mathrm{mm}^{3}\right)$

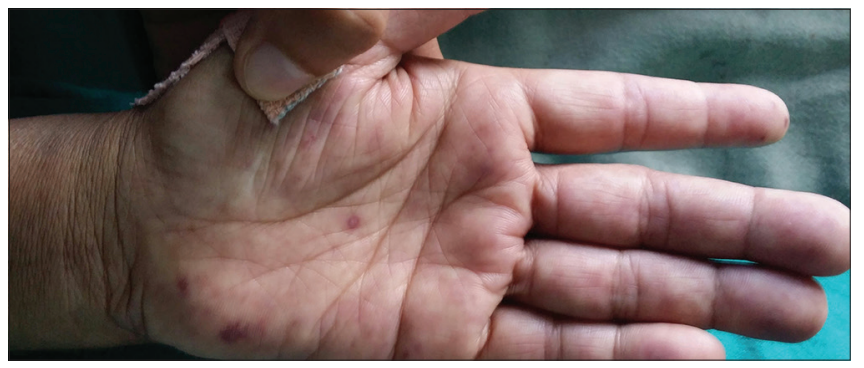

Figure 3: Target-like lesion on the left palm.

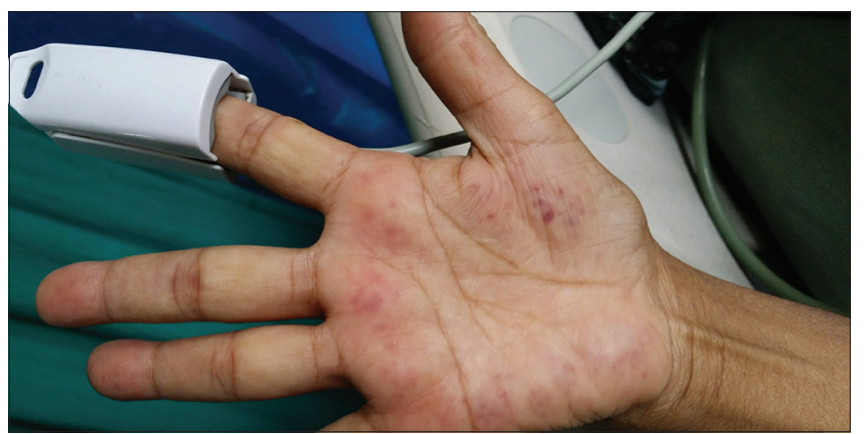

Figure 4: Discrete purpuras on the right palm.

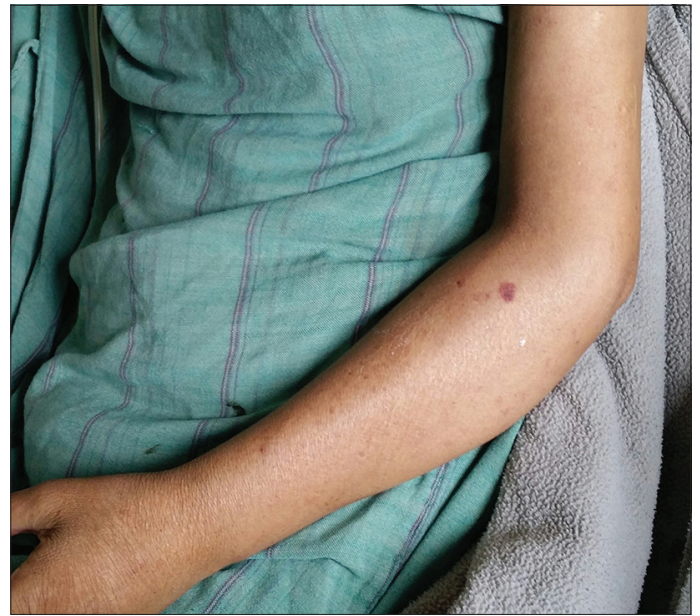

Figure 5: Purpura on her left forearm 
- Differential count - 86\% neutrophils, $10 \%$ lymphocytes and $4 \%$ eosinophils

- Platelets - 65,000/ $\mathrm{mm}^{3}$ (reference value: 1,50,000$\left.4,50,000 / \mathrm{mm}^{3}\right)$

- ESR - $56 \mathrm{~mm}$ in $1^{\text {st }}$ hour

- Peripheral blood smear-normocytic normochromic anaemia with poikilocytosis and anisocytosis

- Urea - $7.9 \mathrm{mmol} / \mathrm{mL}$, Creatinine - $95 \mu \mathrm{mol} / \mathrm{L}$

- $\mathrm{Na}^{+}-141 \mathrm{mEq} / \mathrm{L}, \mathrm{K}^{+}-4.1 \mathrm{mEq} / \mathrm{L}$

- Serology nonreactive for HIV, HBsAg and VDRL

- ECG - sinus rhythm

- Chest X-ray - patchy infiltration with well-defined margins on right upper lobe

- ANA positive in a speckled pattern

- DsDNA - $225 \mathrm{IU} / \mathrm{mL}$ (positive if $>80 \mathrm{IU} / \mathrm{mL}$ )

- Rheumatoid factor - positive

- Anticardiolipin antibody - positive

- Anti-Ro and anti-La antibodies were not performed as these investigations are not available in the hospital and the patient could not afford the investigation (all the investigations that were available in the hospital were provided free of cost)

- Prothrombin time - 17 seconds (Reference value: 12 seconds)

- INR - 1

- Activated partial thromboplastin time -47 seconds (Reference value: $<30$ seconds)

- Routine urine microscopic examination - albumin $3+$

- 24 hours urinary protein $-2.53 \mathrm{~g} /$ day.

Her diagnosis was revised as all three major criteria and one minor criterion (rheumatoid factor positivity) of Rowell syndrome was fulfilled. She also had renal involvement demonstrated by 24-hour urinary protein loss of $2.53 \mathrm{~g} /$ day.

She was subsequently admitted to medical intensive care unit, her antitubercular medications were stopped and she was started on

- Injection hydrocortisone $200 \mathrm{mg}$ IV BD

- Tablet hydroxychloroquine sulfate $200 \mathrm{mg}$ PO BD

- Nasogastric tube placement for enteral feeding

- Removal of hemorrhagic crusts on lips with saline soaked gauze

- Chlorhexidine and lignocaine gel for oral ulcers.

Her condition improved dramatically in the first few days with the decrease in facial swelling and oral lesions. However, on the eighth day of admission, the patient expired suddenly. Her fibrinogen degradation products were $36 \mathrm{mg} / \mathrm{dL}$ (Reference value: $<10 \mathrm{mg} / \mathrm{dL}$ ) and D-dimer level was $1.7 \mathrm{mg} / \mathrm{L}$ (Reference value: $<0.5 \mathrm{mg} / \mathrm{L})$. She had not required ventilation and her saturation was above $95 \%$ in the preceding days. Her cause of death was suspected to be acute shortness of breath secondary to pulmonary embolism. She had not complained of chest pain, frothing from mouth before death. On auscultation of her chest, there were no abnormalities detected. She had not been put on anti-coagulant therapy. Her skin biopsy, which had been planned, could not be carried out.

\section{DISCUSSION}

Our patient fulfilled all the three major criteria of Rowell's syndrome proposed by Zeitouni [3] and one minor criterion, positivity for RA factor. Her lupus erythematosus was presumed to have been induced by anti-tubercular therapy, as she did not manifest any signs and symptoms of lupus erythematosus prior to antitubercular therapy. Our patient's condition was finally diagnosed as Rowell's syndrome secondary to anti-tubercular therapy.

Although Rowell's syndrome commonly afflicts middleaged women, studies have revealed the condition to be present in other groups as well [6,7]. The treatment regimens, which have shown promising results, include prednisolone, azathioprine, antimalarials and ciclosporin $[8,9]$.

Our patient who had been put on injection hydrocortisone and tablet hydroxychloroquine sulfate had initially shown a dramatic improvement of her symptoms. However, she succumbed to death within a few days to shortness of breath secondary to suspected pulmonary embolism. Although her autopsy was not performed, her cause of death was suspected to be pulmonary embolism because of raised fibrinogen degradation products, elevated D-dimer levels, and cardiolipin antibody positivity. She did not have hypothermia $\left(<35^{\circ} \mathrm{C}\right)$ or fever above $38^{\circ} \mathrm{C}$. There was no drop in her arterial oxygen saturation in the last three days prior to her death. Her arterial blood gas analysis showed results within the normal limit. There was no evidence of frothing from her mouth on inspection and no abnormalities detected on auscultation of her chest. Based on these findings and inherent increased risk of pulmonary embolism in lupus erythematosus $[10,11]$, her cause of death was suspected to be a pulmonary embolism. 


\section{www.odermatol.com}

\section{CONCLUSION}

We recommend monitoring bleeding and clotting parameters in all patients with Rowell's syndrome and lupus erythematosus and initiating anticoagulant therapy to prevent thromboembolism.

\section{REFERENCES}

1. Scholtz M. Lupus erythematosus acutus disseminates haemorrhagicus. Arch Derm Syphilol. 1992;6:466.

2. Rowell NR, Beck JS, Anderson JR. Lupus erythematosus and erythema multiformae-like lesions: A syndrome with characteristic immunological abnormalities. Arch Dermatol. 1963;88:176-80.

3. Zeitouni NC, Funaro D, Cloutier RA, Gagne E, Claveau J. Redefining Rowell's syndrome. Br J Dermatol. 2000;142:343-6.

4. Durmazlar SPK, Oktay B, Eren C, Eskiogly F. So-called Rowell's Syndrome: Report of a Case. J Turk Acad Dermatol. 2009;3:92201c.

5. Dalle Vedove C, Simon JC, Girolomoni G. Drug-induced lupus erythematosus with emphasis on skin manifestations and the role of anti-TNF-alpha agents. J Dtsch Dermatol Ges. 2012;64:2677-86.

6. Bhat RY, Varma C, Bhatt S, Balachandran C. Rowell syndrome. Indian Dermatol Online J. 2015;5:33-5.
7. Lee A, Baltra P, Furer V, Cheung W, Wang N, Franks A. Rowell syndrome (systemic lupus erythematosus + erythema multiforme). Dermatol Online J. 2009;15:1.

8. Aydogan K, Karadogan S, Balaban Adim S, Tunali S. Lupus erythematosus associated with erythema multiformae: Report of two cases and review of the literature. J Eur Acad Dermatol Venereol. 2005;19:621-7.

9. Muller CS, Hinterberger LR, Vogt T. Successful treatment of Rowell syndrome using oral cyclosporine A. Int J Dermatol. 2011;50:1020-2.

10. Chung WS, Lin CL, Chang SN, Lu CC. Kao CH. Systemic lupus erythematosus increases the risks of deep vein thrombosis and pulmonary embolism: a nationwide cohort study. J Thromb Haemost. 2014;12:452-8.

11. Avina-Zubieta JA, Lacaille D, Sayre EC, Kopec J, Choi HK and Esdaile JM. Risk of pulmonary embolism and deep vein thrombosis in systemic lupus erythematosus: a population-based cohort study. Arthritis Res Ther. 2012;14:A53.

Copyright by Bibush Amatya, et al. This is an open access article distributed under the terms of the Creative Commons Attribution License which permits unrestricted use, distribution, and reproduction in any medium, provided the original author and source are credited.

Source of Support: Nil, Conflict of Interest: None declared. 